\title{
MiR-142-3p regulates Cardiovascular System during zebrafish development
}

\author{
Ziyad Tariq Muhseen ${ }^{1, *}$, Nibras Najm Abbood ${ }^{1,2}$ \\ ${ }^{1}$ Key Laboratory of Molecular Biophysics, Department of Biotechnology, College of Life Science and Technology, Huazhong University of \\ Science and Technology, Wuhan, Hubei, China \\ ${ }^{2}$ Marine Sciences Centre, University of Basra, Basra, Iraq
}

\section{Email address:}

Ziyad.tariq82@gmail.com (Z. T. Muhseen),genetechnology2012@gmail.com (N. N. Abbood)

\section{To cite this article:}

Ziyad Tariq Muhseen, Nibras Najm Abbood. MiR-142-3p Regulates Cardiovascular System during Zebrafish Development. Cell Biology. Vol. 2, No. 6, 2014, pp. 49-59. doi: 10.11648/j.cb.20140206.11

\begin{abstract}
MicroRNA-142-3p (miR-142-3p) is considered to play key role in many cellular processes, such as proliferation, differentiation and apoptosis by inhibiting target gene expression. The transcriptional regulation of cardiovascular development requires precise spatiotemporal control of gene expression. We are beginning to understand the functions of miRNA-142-3p played during essential biological processes especially erythropoiesis. Here, we overview the recent findings on miRNA-142-3p regulation in erythropoiesis development and report the latest advances in understanding their function by unveiling their mRNA targets. Further analysis of miRNA-142-3p function during erythropoiesis development will allow us to determine the potential for novel miRNA-142-3p -based therapeutic strategies. Haematopoiesis is an active process by which peripheral blood families are developed. It is a process strongly regulated by many essential and unessential factors, including signalling molecules and transcriptional factors. However, the epigenetic regulation of haematopoiesis regulation via microRNAs (miRNAs) remains partially understood. Here, in this study we show that miRNA-142-3p participates in the haematopoiesis regulation and vascular development of the early stages of erythropoiesis during Zebra fish early developmental stages. Overexpression inhibited the primitive haematopoiesis, characterized by a significant reduced number and limitation of erythropoiesis cells and myeloid expansion significantly reduced expression of runx1, c-myb, mpo-1, Scl and pu.1, Gata1, Gata2 and significantly reduced o-dianisidine staining of haemoglobin. Systematically, miR-142-3p regulates haematopoiesis by repressing expression of meis1 involved in haematopoiesis. We performed knock-down analysis of the meis 1 gene to check and demonstrate that whether meis1-EGFP is related with our miRNA. Co-injection-confirmation at 24 and 48 hpf pictures analyses showed that overexpression of miR-142-3p repressed expression of meis1. Bioinformatics analysis predicts a target binding sequence for miR-142-3p at the 3-UTR of meis1. Deletion of the miR-142-3p target sequence eliminated the repression of meis 1 expression mediated by miR-142-3p. These findings create miR-142-3p as a novel miRNA that regulates haematopoiesis and cardiovascular development by repressing expression of meis1.
\end{abstract}

Keywords: Mir-142-3p, Meis1, Hematopoiesis, Cardiovascular, Zebrafish

\section{Introduction}

MicroRNA-142-3p are a large class of evolutionarily conserved small non-coding RNAs, typically 20-26 nucleotides (nt) in length , that primarily function post-transcriptional by interacting with the 3' un translated region (UTR) of specific target mRNAs in a sequence-specific manner[1]. Haematopoiesis is a highly regulated, life-long, multi-stage process by which blood cell lineages are generated [1-4]. Haematopoiesis in vertebrates is occurring in two consecutive waves, including primitive and definitive haematopoiesis [5-7]. The understanding of the genetic regulation in haematopoiesis is essential because disorder in haematopoiesis results in diseases such as thrombocytopenia, anaemia, lymphoma, and leukaemia [8, 9]. Like to most vertebrates, zebra fish embryonic haematopoiesis initiates from the lateral plate mesoderm (LPM), which gives growth to hemangioblasts, cardiac and other tissues [8]. Hemangioblasts are multi-potential progenitors that generate pluripotent self-renewing hematopoietic stem cells (HSCs) and vascular endothelial cells. HSCs can subsequently produce erythrocytes, 
myelocytes, and other blood cells $[10,11]$. Primitive haematopoiesis, which creates erythrocytes and macrophages, begins in the blood island of the yolk sac at the embryonic stage of about 12 hour's post-fertilization (hpf) in zebra fish. But definitive haematopoiesis, which produces HSCs that give rise to all blood lineages, begins in the aorta-gonad-mesonephros (AGM) region at about $36 \mathrm{hpf}$ in zebra fish [8, 12].

Previous to the differential expression of either myeloid or erythroid genes in these Distinct precursor pools, both regions express the transcription factor gene scl. The stem cell leukaemia (scl) gene acts upstream of pu.1, which control the common myelo-erythroid progenitors (MEPs) to determine erythroid versus myeloid fate [13-15]. In addition to a variety of protein-coding mRNAs, accumulating evidence suggests that microRNAs (miRs, i.e. short, 22-nucleotide non-coding RNAs) regulate haematopoiesis by acting as post-transcriptional modulators of gene expression and exerting significant effects on cell growth, development, and differentiation [16, 17]. Several miRs have been reported to regulate haematopoiesis. Deregulation of miR-155, miR-451, and miR-320 has been shown to result in blood diseases [18-20]. Zebra fish miR-142-3p is specifically expressed in the hematopoietic tissue, co-transcribed with miR-451, and shares the same nucleotide sequence with miR transcripts from other species. In this study, through ectopic expression of miR-142-3p in zebra fish embryos, we show that miR-142-3p regulates definitive haematopoiesis as well as vascular development by targeting meis1, which provides significant insights into a novel miR-based molecular signal pathway for haematopoiesis and vascular development.

\section{Materials and Methods}

\subsection{Fish Maintenance and Embryos Collection}

First, Wild-type zebra fish (Danio rerio) (AB type) were cultured in the fish culture laboratory according to the zebra fish Book [21] and maintained at $28.5^{\circ} \mathrm{C}$ under standard conditions as described previously [22]. To prevent pigmentation, embryos were raised and maintained at $28.5^{\circ} \mathrm{C}$ in "egg water" consisting of $0.003 \%$ 1-phenyl-2-thiourea (PTU, Sigma) after 12 hpf [21]. Studies using zebra fish were approved by the Animal Care and Use Committee of Chinese Academy of Science, Institute of Hydrobiology (Wuhan). Embryos were obtained from artificial zebra fish eggs and sperm were collected from the fish culture laboratory. After fertilization and hatching, larvae were transferred to small glass aquaria and kept for 3 days and then preserved in the TRIzol ${ }^{\circledR}$ reagent (Invirogen, USA) in $-70^{\circ} \mathrm{C}$ refrigerator pending for total RNA isolation [21].

\subsection{Plasmid Construction}

The 3-UTR of meis1 was amplified by PCR from a cDNA library prepared from 24 hpf zebra fish embryos and cloned between the Spe I-Hind III sites of the pMIR -REPORT Luciferase vector [23] resulting in pMIR-meis1-3-UTR (wt). The ORF of EGFP was cloned between the BamH I-EcoR I sites of the pCS2+vector [24, 25], whereas the meis1-3-UTR was cloned between the EcoR I-Xho1 I sites of the PCS2 vector. The primers used in this study are listed in Table 1. All constructs were validated by restriction enzyme digestion and direct DNA sequencing analysis.

Table 1. Primer sequences for meisl are described.

\begin{tabular}{lll}
\hline (Gene Bank Accession No.) & Primer name & Sequence (5'-3') \\
\hline NM_131893.1 & ImR-F2 EcoR1 & GTAAGAATTCCCTTCTAGTTAACCAATCGC \\
NM_131893.1 & ImR-R2 Xho-1 & GAGGCTCGAGTCTATTAGAAACAACATAATATG \\
\hline
\end{tabular}

\subsection{Bioinformatics Analysis}

The direct targets and binding sites for miR-142-3p were predicted using several online programs, including TargetScan (http://www.targetscan.org/) and EIMMO (http://www.mirz.unibas.ch/ElMMo2/) [23, 25]. Meis1 was predicted to be the direct downstream target gene of miR-142-3p by all the programs.

\subsection{Preparation of cDNA}

cDNA was synthesized by reverse transcription [26] from
$1 \mu \mathrm{g}$ of total RNA using the Rever Tra Ace enzyme (TOYOBO, Japan), dNTPs and Oligo [27] 20 RT primer (TOYOBO, Japan). The RT reaction was performed at $37^{\circ} \mathrm{C}$ for $60 \mathrm{~min}$ followed by $98^{\circ} \mathrm{C}$ for $5 \mathrm{~min}$.

\subsection{MiRNA Mimics and Preparation}

Mimics and preparation of mRNAMiR-142-3p mimics and scrambled control miRNA mimics were purchased from RioboBio (RiboBio, China).

Table 2. PrimersequencesformiR-142-3p are described.

\begin{tabular}{ll}
\hline Primer name & Sequence (5'-3') \\
\hline miR-142-3p -primer1 & 5'5-GCGACTGTTCCTCTCTTCCTCTGTGTGTGTGTGTGTAGTCGCAACCATAAA-3' \\
miR-142-3p -primer2 & 5'-CTCCTGTAGTGTTTCCTAC-3' \\
miR-142-3p- primer3 & 5'-GACTGTTCCTCTCTTCCTC-3' \\
\hline
\end{tabular}

\subsection{Microinjection}

Injected with $1 \mathrm{nl}$ of DNA or RNA sample and each sample was injected with 300 embryos. Microinjection was performed with the aid of an Eppendorf 5242 (Germany) 
micro injector. Glass needles were prepared using a Narishige PN-30 (Japan) pipette puller and Flaming/Brown Micropipette Puller PN-97 (USA). The microinjected eggs were allowed to develop in Danieau ${ }^{\text {ee }}$ buffer $(58 \mathrm{mM} \mathrm{NaCl}$, $0.7 \mathrm{mM} \mathrm{KCl}, 0.4 \mathrm{mM} \mathrm{MgSO} 4,0.6 \mathrm{mM} \mathrm{Ca}(\mathrm{NO} 3) 2,5 \mathrm{mM}$ Hepes, $\mathrm{pH} 7.6)$ and cultured in incubator at $28.5^{\circ} \mathrm{C}$ until 3 dpf.

\subsubsection{Microinjection of Synthetic Mir-142-3p}

Microinjection of synthetic miR-142-3p duplexes and mRNA One to two cell stage fertilized embryos were microinjected with 1-2 $\mathrm{nl}$ of synthetic miR-142-3p duplexes $\left(10 \_\mathrm{M}\right)$ and control mimics $\left(10 \_\mathrm{M}\right)$ as described [28].

\subsubsection{Microinjection Confirmation Meis1-Egfp}

To confirm whether our meis1-EGFP will exert its effect on haematopoiesis, we have injected meis1-EGFP with concentration $0.91 \mathrm{ng} / \mu \mathrm{L}(0.91 \mu \mathrm{L}$ of meis 1 -egfp with $2.09 \mu \mathrm{L}$ of RNase free water). It was microinjected into embryos at the single cell stage. Microinjection volumes were estimated at around $1 \mathrm{nl}$ per embryo. And we have checked the GFP (green fluorescent protein) at 6 somite stage, 24 and 48 hours post fertilization (Figure 1), the result was positive for meis1-EGFP.

$\begin{array}{lll}\text { A } & \text { miR-142-3p targeting sequences in the 3-UTR of mei } \\ \text { Has } & \text { AUACGUACAUUCCUGGACACCCUCACCACCCAA } & 179 \\ \text { Mmu } & \text { AUACGUACAUUCCUGGACACCCUCACCACCCC } & 170 \\ \text { ptr } & \text { AUACGUACAUUCCUGGACACCCUCACCACCCAA } & 170 \\ \text { Rno } & \text { AUACGUACAUUCCUGGACAUCCUCACCACCCC } & 170 \\ \text { Mml } & \text { AUACGUACAUUCCUGGACACCCUCACCACCCAA } & 170 \\ \text { Bta } & \text { AUACGUACAUUCCUGGACACCCUCACCACCCAA } & 170 \\ \text { Mdo } & \text { AUACCUACAUUCCUGGACACCCUCACCACCCAA } & 170 \\ \text { Xtr } & \text { AUACCUACAUUCCGGACACCAUCACCACCCAG } & 170\end{array}$

\section{B}

hsa-miR-142-3p
mmu-miR-142-3p
rno-miR-142-3p
ptr-miR-142-3p
mml-miR-142-3p
bta-miR-142-3p
mdo-miR-142-3p
xtr-miR-142-3p

Figure 1. Bioinformatic analysis identified miR-142-3p targeting sequences in the 3-UTRof meis1 from various vertebrate species. (A) 3-UTR sequences of meis1 containingthe miR-142-3p targeting sites ('seeds') were aligned among humans (Hsa), mouse (Mmu), chimpanzee(Ptr), rat (Rno), Rhesus (Mml), Cow (Bta), Opossum (Mdo), Frog (Xtr)and zebrafish (Dre).Numbers indicated nucleotide positions targeted by the seed sequence of miR-142-3p. .The zebrafish meis1 3-UTR contained a pair of target sequences for miR-142-3p . (B) The identity of the sequence of mature miR-142-3p among different species.

\begin{abstract}
UAUGUAUGAAGAAAUGUAAGGU
UAUGUAUGAAGAAAUGUAAGGU

AUGUAUGAAGAAAUGUAAGGU

AUGUAUGAAGAAAUGUAAGGU
\end{abstract}

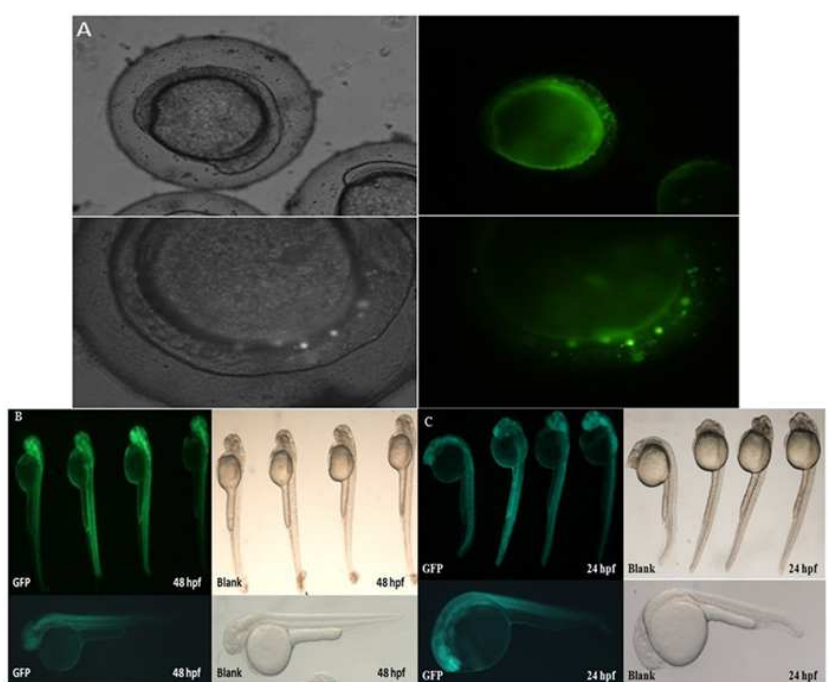

Figure 2. In vivo reporter Microinjection confirmation meis1-egfp. (A) Confirmation of pcs2+-egfp by microinjection and egfp observation at 6 somite stage show the egfp observation In both blank and green fluorescent pictures $(B, C)$ The egfp observation In both blank and green fluorescent pictures at 24 and $48 \mathrm{hpf}$.

\subsubsection{Microinjection of Meis1-Pcs2+-EGFP mRNA}

After natural fertilization, the zygotes were dechorionated by $200 \mu \mathrm{l}$ of $10 \mathrm{mg} / \mathrm{ml}$ Pronase (Roche, USA) and followed by three times wash with water and three times wash with Danieau ${ }^{\text {ee }} \mathrm{s}$ buffer $(58 \mathrm{mM} \mathrm{NaCl}, 0.7 \mathrm{mM} \mathrm{KCl}, 0.4 \mathrm{mM}$ MgSO4, 0.6 mM Ca(NO3)2, 5 mM Hepes, pH 7.6).

\subsection{RT-PCR (Reverse-Transcription PCR) Analysis of Meis1}

Total RNA of 50 zebra fish embryos from 3-Day post fertilization of wild type zebra fish were extracted using TRIzol® reagent (Invitrogen, USA) according to the manufacturer's instructions. All RNA samples were treated with RNase-Free DNase (Promega, USA), to remove genomic DNA contamination according to manufacturer's protocol. PCR was performed to study the expression of meis1. Gene Bank accession number and primer sequences are described in (Table.1). PCR was carried out in a $50 \mu \mathrm{L}$ reaction volume containing $5 \mu \mathrm{L}$ of $10 \times$ PCR buffer, $1.5 \mu \mathrm{L}$ of each primer, 5 $\mu \mathrm{L}$ of $2 \mathrm{mM}$ dNTP, $5 \mu \mathrm{L}$ of $\mathrm{mM} \mathrm{MgCl} 2,1 \mu \mathrm{L}$ of $\mathrm{Kod}_{+} \mathrm{Neo}$ (1unit $/ \mu \mathrm{L}$ ), $2 \mu \mathrm{L}$ of cDNA solution, $31 \mu \mathrm{L}$ of D.W . The PCR reaction consists of a preheating at $94^{\circ} \mathrm{C}$ for $2 \mathrm{~min}$, denaturation at $98^{\circ} \mathrm{c}$ for $10 \mathrm{sec}(35$ cycles $)$, annealing at $55^{\circ} \mathrm{c}$ for $30 \mathrm{sec}$ ( 35 cycles), extension at $68^{\circ} \mathrm{c}$ for $50 \mathrm{sec}$ ( 35 cycles) with a final elongation step of $10 \mathrm{~min}$ at $68^{\circ} \mathrm{C}$. The products were run on $1 \%$ agarose gels stained with ethidium bromide $(0.5 \mu \mathrm{g} / \mathrm{mL})$ and amplified bands were visualized by ultraviolet trans-illumination and semi-quantified by Glyko Bandscan software (Novato, CA). A molecular weight marker DNA (DL2000; 100ng/l conc.) was run on left side of the gel.

\subsection{Real-Time Quantitative PCR Analysis of U6 Transcript and Target genes Analysis of Mir-142-3p}

QRT-PCR assays were performed for measurement of the expression levels of primary, precursor and mature miRNAs. 
Fertilized zebra fish zygotes were Collected in different stages : $\{1$ cell, 16cell, 512 cell, Oblong, 5.3h (50\%- epiboly), 3 somite, 6somite, 1 day and 2 day\}.

Each embryo was wild type and we keep it in Trizol, cDNA samples from the manipulated zebra fish embryos at 2-cell stage and $3 \mathrm{dpf}$ or $1 \mathrm{dpf}$ were analysed with real-time quantitative PCR (Q-PCR).

Real-time PCR was performed on an ABI PRISM $® 7000$ Sequence Detector (Applied Bio-systems, Inc.USA) according to the manufacturer's instructions. Reactions were performed in a $20 \mu \mathrm{l}$ volume with $150 \mathrm{ng}$ of cDNA, $0.2 \mu \mathrm{M}$ primers and $10 \mu \mathrm{l}$ of SYBR® Green Real-time PCR Master Mix (TOYOBO, Japan). The primer pairs were shown in (table 3).

Table 3. The primer pairs for the real-timePCR master mix.

\begin{tabular}{ll}
\hline Primer name & Sequence (5'-3') \\
\hline miR-142-3p -primer1 & 5'-GCGACTGTTCCTCTCTTCCTCTGTGTG \\
miR-142-3p -primer2 & TGTGTGTGTAGTCGCAACCATAAA-3' \\
miR-142-3p - '- CTCCTimer3 & 5'-GACTGTTCCTCTCTCTTCCTC-3' \\
U6 snRNA forward & 5'-CTCGC TTCGG CAGCA CA-3' \\
U6 snRNA reverse & 5'-AACGC TTCAC GAATT TGCGT-3' \\
\hline
\end{tabular}

All reactions were run using the following conditions: 1 min denaturation step followed by 40 cycles with a $95{ }^{\circ} \mathrm{C}$ denaturation for $15 \mathrm{~s}, 55{ }^{\circ} \mathrm{C}$ annealing for $15 \mathrm{~s}$, and $72{ }^{\circ} \mathrm{C}$ extension for $45 \mathrm{~s}$. Detection of the fluorescent product was carried out at the end of the $72{ }^{\circ} \mathrm{C}$ extension period. The data (Ct value), obtained from the software (7000 system SDS software) was transferred to Windows Excel (Microsoft co.) sheet and fold-change was calculated using 2- $\Delta \Delta \mathrm{Ct}$ method, Results were normalized with U6 RNA as the calibrator For measurement of the primary and precursor miRNA expression. The RT process conditions and the PCR process conditions are all given in (Table.3).

\section{9. mRNA Synthesis of Meis1-EGFP: Capped Transcription Reaction Assembly}

We have used $20 \mu \mathrm{L}$ of mixture contain $10 \mu \mathrm{L}$ of $2 \mathrm{XNTP} / \mathrm{CAP}, 2 \mu \mathrm{L}$ of $10 x$ reaction buffer, $6 \mu \mathrm{L}$ of linear template DNA(meis1-pcs2+-EGFP), $2 \mu \mathrm{L}$ of enzyme mix ( before mix we have shaken all of it until they became completely in solution, and 2XNTP/CAP, linear template DNA(meis1-pcs2+-EGFP), enzyme mix have stored on ice but 10xreaction buffer was keeping in room temperature (because of The spermidine in the 10X Reaction Buffer can co precipitate the template DNA if the reaction is assembled on ice) and then it was mixed with pipette gently and centrifuge a little, and incubated at $37^{\circ} \mathrm{c}$ for 2 hours in water bath, after that we added $1 \mu \mathrm{L}$ of Turbo DNase and mix it and incubated at $37^{\circ} \mathrm{c}$ for $15 \mathrm{~min}$. and then added $205 \mu \mathrm{L}$ of nuclease free water and mix it. Then added $25 \mu \mathrm{L}$ of NH4AC and mix it, then we mixed $125 \mu \mathrm{L}$ of phenol (PH 4.5) and $125 \mu \mathrm{L}$ of CH3CL3 and added it and mix it vigorously for $15 \mathrm{sec}$. And then incubated at room temperature for 2-3 minutes then centrifuge at $4^{\circ} \mathrm{c}$ for 15 minutes with $12000 \mathrm{rpm}$ and then we transferred the upper aqueous to a new ependrop tube and added $250 \mu \mathrm{L}$ of
Isopropanol (isopropyl alcohol) and mixed well and chilled the mixture at $-20^{\circ} \mathrm{c}$ for 1 hour or at $-70^{\circ} \mathrm{c}$ for 30 minutes and then centrifuge it at $4^{\circ} \mathrm{c}$ for 30 minutes with $12000 \mathrm{rpm}$. Then discarded the aqueous and dried the pellet at room temperature for 5-10 minutes and then added $11 \mu \mathrm{L}$ of nuclease free water and stored at $-70^{\circ} \mathrm{c}$.

\subsection{Total RNA Isolation}

To isolate total RNA from zebra fish, we have used Trizol reagent (Invirogen, USA). In the laboratory the temperature was around $22^{\circ} \mathrm{C}$ we homogenized tissue samples (zebra fish embryos), it was around 50 embryos with $0.8 \mathrm{ml}$ of Trizol in one epindrop tube $(1.5 \mathrm{ml}$ tube) and then incubated at 15 to $30^{\circ} \mathrm{c}$ for 5 minutes to permit the complete dissociation of nucleoprotein complex. And added $0.2 \mathrm{ml}$ of chloroform and shacked it vigorously by hand for $15 \mathrm{sec}$ and incubated it at $15-30^{\circ} \mathrm{c}$ for $2-3$ minutes followed by centrifugation at $4^{\circ} \mathrm{c}$ for 15 minutes with $12000 \mathrm{x}$ g. After centrifuge the mixture separated in to a lower red phenol-chloroform phase and a colourless upper aqueous phase. RNA was in the aqueous phase, we transferred RNA from aqueous phase to a fresh tube and added $0.5 \mathrm{ml}$ of isopropyl alcohol to precipitate the RNA from the aqueous phase, incubated at $15-30^{\circ} \mathrm{c}$ for 10 minutes and centrifuged at $4^{\circ} \mathrm{c}$ for 10 minutes with $12000 \mathrm{x} \mathrm{g}$ and then discarded the supernatant and added $1 \mathrm{ml}$ of $75 \%$ ethanol to wash the RNA pellet, mixed the sample by using vortex and centrifuge again it at $4^{\circ} \mathrm{c}$ for 4 minutes with 7500x $\mathrm{g}$ and then we dried the RNA pellet for 5 minutes and then added RNase-free water to dissolve RNA, incubated it at $55-60^{\circ} \mathrm{c}$ for 10 minutes then stored it at $-70^{\circ} \mathrm{c}$.

\subsection{Competent Cells Preparation}

We have done preparation for competent cell to use it for transformation of meis1-3UTR-pcs2+-EGFP. So in AIRTHCH hood we have cultured $2-5 \mu$ of stock E. coli in (L.B agar media without antibiotic Ampicillin A+ or Kanamycin $\mathrm{K}+$ ) in Petri dish overnight. And With small tip we have taken one colony and add it to $5 \mathrm{ml}$ of L.B media without $\mathrm{A}+$ or $\mathrm{K}+$ in $10 \mathrm{ml}$ tube and shaked it with shaker at $37^{\circ} \mathrm{C}$ with $80-100 \mathrm{rpm}$ for 12 hours and then Added $1 \mathrm{ml}$ of this media to $100 \mathrm{ml} \mathrm{L.B}$ media without $\mathrm{A}+$ or $\mathrm{K}+$ for 3 hours. During these 3 hours (each 90 minutes we checked $\mathrm{OD} / 600)(\mathrm{OD} / 600=0.4-0.6)$, after that put it in ice box and put CACL2 also in ice box). Divided it into 2 tubes $(50 \mathrm{ml}$ tubes) and centrifuge at $4^{\circ} \mathrm{c}$ for 10 minutes $/ 3000 \mathrm{rpm}$ and then discarded the liquid and added to each tube 6-10 ml (CACL2 $0.1 \mathrm{~mol} / \mathrm{L}$ ) and mix it well and put it in ice box for 10 minutes. Centrifuge at $4^{\circ} \mathrm{c}$ for 10 minutes $/ 3000 \mathrm{rpm}$ and then discarded all the liquid and added 4ml (CACL2 0.1 $\mathrm{mol} / \mathrm{L}$ ) for each tube, Added for each tube $1.2 \mathrm{ml}$ of Glycerol and mix it very well. And then divided it to ependrop. tubes (each $100 \mu \mathrm{l}$ in $1.5 \mathrm{ml}$ Ep. tube) and Keep it at $-80^{\circ} \mathrm{c}$.

\subsection{O-Dianisdine Staining}

O-Dianisidine staining was used to detect the distribution of hemoglobin of zebrafish by o-dianisdine. It was carried 
out as described previously [28, 29]. First, we started with preparing staining solution containing $0.2 \%$ benzidine (Sigma), sodium acetate (0.01 M, pH 4.5) and ethanol (40\%). Prior to use $1 / 10 \mathrm{~V}$ of $3 \%$ hydrogen peroxide was added. Embryos were collected in $2 \mathrm{ml}$ micro eppendorf tubes with staining solution that covers all embryos. The embryos were stained for 15-20 min at room temperature to avoid light and inspected at every 3- $5 \mathrm{~min}$. The embryos were then imaged under a microscope and analysed by measuring the corresponding stained areas of the embryos with Image.

\subsection{Whole-Mount in Situ Hybridization (WISH)}

Whole-mount in situ hybridization (WISH) was carried out using Dig-labeled mRNA antisense probes as described previously [22, 30]. Embryos at 12 hpf, 24 hpf, 36 hpf or 48 hpf were dechorionated fixed in $4 \%$ paraformaldehyde (PFA) / phosphatebuffer saline (PBS) overnight at $4{ }^{\circ} \mathrm{C}$, washed twice in PBS at room temperature and then maintained in absolute methanol at $-20^{\circ} \mathrm{C}$ until use. Embryos were rehydrated by successive dilutions using methanol/PBS (75, 50 and 25\%). Then the embryos were treated with proteinase $\mathrm{K}\left(10 \_\mathrm{g} / \mathrm{ml}\right)$ at room temperature, re-fixed with $4 \%$ PFA/PBS for $20 \mathrm{~min}$, washed several times with PBST (PBS $+0.1 \%$ Tween-20) and pre-hybridized for 2 hour with the hybridization solution without the probe. Then the digoxin-labelled probe was added (1 ng/ _ 1 ) and incubated overnight at $65^{\circ} \mathrm{C}$. Hybridization signals were detected using anti-digoxigenin-AP (Roche, Mannheim, Germany) and by staining with BCIP/NBT(Promega). The probes used for WISH has been described previously [22].

\subsection{Image Acquisition}

Post-injection phenotypes were observed at different time points of the development stages. Embryos were photographed at 40 magnifications using an Olympus SZX12 microscope with a Nikon Digital Sight DS-SMc digital camera (Nikon, Japan). The dorsal and lateral views of the 0 $\mathrm{dpf}, 1 \mathrm{dpf}$ and $2 \mathrm{dpf}$ larvae were photographed at 40 magnifications using an Olympus SZX12 stereomicroscope and a Nikon Digital Sight DS-SMc digital camera (Nikon, Japan). And high-power images with a Zeiss Stereo lumar.V12 microscope with a Zeiss AxioCam Mic 01746 digital camera and AxioVision HRC/Neolumrs1.5X FWO. Green fluorescence machine (illumination system) KL2500LCD, HBO1000 software (Zeiss, Welwyn Garden City, United Kingdom). Identical modifications and adjustments were applied to all the images in the same experiment [31]. Pictures were processed using Adobe Photoshop software.

\subsection{Statistical Analysis}

All experiments were repeated at least three times. The data were presented as mean \pm SD. Statistical analysis was performed during the student's t-test with Spass13.0 software. A $\mathrm{P}$ value of $<0.05$ was considered to be statistically significant.

\section{Results}

\subsection{Bioinformatic Analysis Identifies Meis1 as a Potential Downstream Target of miR-142-3p}

Bioinformatics analysis with Target Scan, EIMMo, Microcosm Targets Version 5, and PicTar software programs was used to identify potential downstream targets regulated by miR-142-3p. We analysed the 3-untranslated region (3-UTR) of meis1 for potential target sites for miRNAs. We found that the meis1 3-UTR of zebra fish contained two adjoining target sites for miR-142-3p (Fig.1A).

The miR-142-3p -binding site was highly conserved during evolution among the humans (Hsa), mouse (Mmu), chimpanzee(Ptr), rat (Rno), Rhesus (Mml), Cow (Bta), Opossum (Mdo), Frog (Xtr)and zebrafish (Dre).

\subsection{Mir-142-3p is Required for Erythropoiesis Limitation and Myeloid Expansion in Zebrafish}

To demonstrate the physiological function of miR-142-3p -mediated control of meis 1 expression, we analysed the effects of miR-142-3p on haematopoiesis. Previous reports have shown that meis1 is required for primitive and definitive haematopoiesis in zebra fish embryos [32-34].

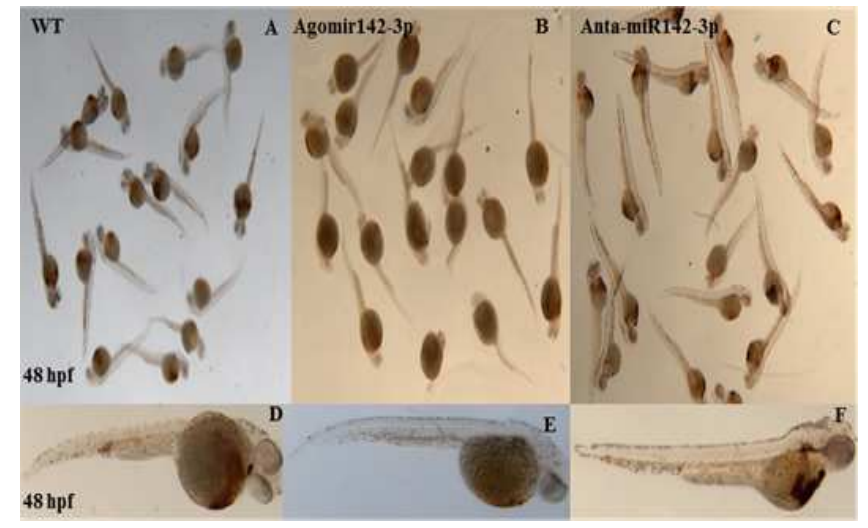

Figure 3. The o-staining result of miR-142-3p over expression at $48 \mathrm{hpf}$ shows a severe blood formation blocking $(A, D)$ wt control-lateral view $(B, E)$ agomir142-3p injected embryos lacked staining completely-lateral view (C,F) antagomiR-142-3p injected embryos knockdown agomir142-3p expression to show increase in hematopoiesis, compared with lateral view.

Therefore, we hypothesized that miR-142-3p was also involved in haematopoiesis by repressing meis 1 . We analysed erythropoiesis by o-dianisidine staining of haemoglobin (Fig. 3). Ectopic expression of miR-142-3p dramatically reduced the number of circulating blood cells in 32 hpf embryos and 2 dpf embryos. The reduced number of circulating blood cells of miR-142-3p injected embryos (with Agomir142-3p and Antagomir142-3p) prompted us to test whether miR-142-3p plays an important role in haematopoiesis during early zebra fish development. To examine the requirement of the miR-142-3p transcript during haematopoiesis for the expression of miR-142-3p in both primitive and definitive haematopoiesis, we first tested whether depletion of miR-142-3p affects the number of erythroid cells using o-dianisidine staining. In control 
embryos, haemoglobin-positive cells were found soon after circulation started At 36 hpf (Figure 3. A, D, C, F) whereas miR-142-3p injected embryos lacked staining completely (Figure 3. B, E).

Moreover, the intensity of endothelial signal in the dorsal aorta and cardinal vein areas were decreased in embryos with injection of miR-142-3p compared to the wild type. Conversely, miR-142-3p injected embryos exhibited an $85 \%$ decrease in the number of erythroid cells at $48 \mathrm{hpf}$ and at 72 hpf. Consistently, miR-142-3p injected embryos had the same lack of red blood cells (Figure 6).

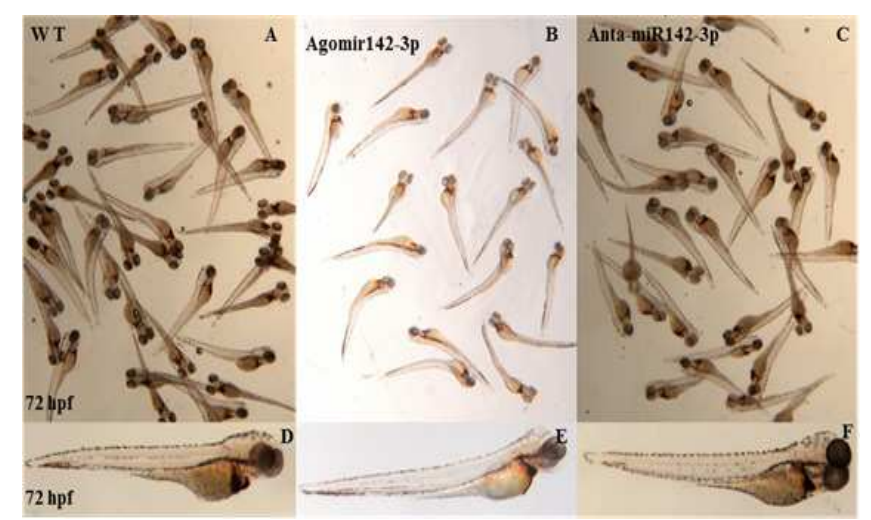

Figure 4. the o-staining result of miR-142-3p over expression at $72 \mathrm{hpf}$ shows a severe blood formation blocking-Ventral view $(A, D)$ wt control $(B$, E,C,F) miR-142-3p injected embryos lacked staining.

Furthermore, in control embryos at 72 hpf erythrocytes were robustly stained with o-dianisidine and were found to be prominent in the ducts of Cuvier over the yolk sac (Figure 4).

A possible defection in primitive erythropoiesis attributed to the slower circulation in miR-142-3p treated embryos was excluded by analysing embryos that completely lacked circulation. In control embryos at $96 \mathrm{hpf}$, erythrocytes were very robustly stained with o-dianisidine and were found to be prominent in the ducts of Cuvier over the yolk sac (Figure 5). Conversely, miR-142-3p injected embryos exhibited a $40 \%$ decrease in the number of erythroid cells at $96 \mathrm{hpf}$.

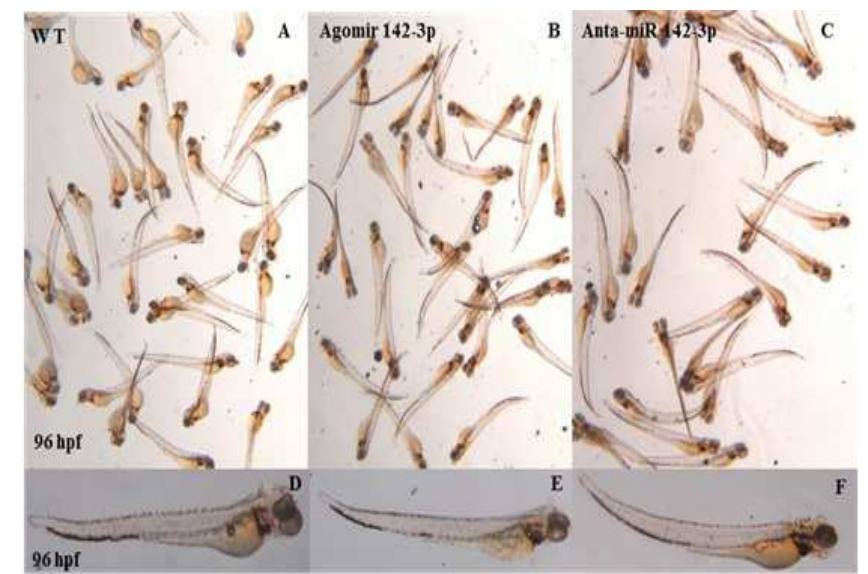

Figure 5. The o-staining result of miR-142-3p over expression at $96 \mathrm{hpf}$ (lateral view) shows a good blood formation in the injected embryo $(A, D)$ WT control (B, E,C, F) miR-142-3p injected embryos lacked staining.
The balance between progenitor and differentiated blood cell populations is crucial for the long-term maintenance of functional blood cell lineages. Progenitor cells maintain this balance by choosing one of several alternate paths: self-renewal through proliferation, commitment to differentiation, and senescence or cell death.

Between the $48 \mathrm{hpf} \& 72 \mathrm{hpf}$ pictures we can see that the miR-142-3p effective is less in the $72 \mathrm{hpf}$ than in $48 \mathrm{hpf}$ suggested that: (i) The miR-142-3p has a short effective term, during the first $48 \mathrm{hpf}$ it show a high remarkable blocking for the blood cell formation in the heart and blood vessels but after that its show a little effect. (ii) The cardio vascular system can recover from the effect of the miR-142-3p. This demonstrates that mir-142-3p is an essential regulator of primitive erythropoiesis in zebra fish.

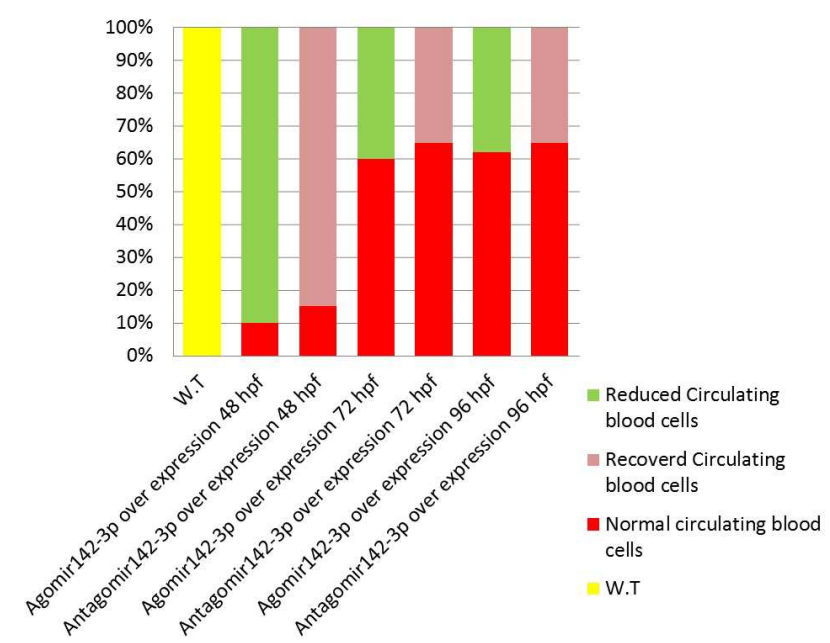

Figure 6. The 48, 72, 96 hpf o-staining result percentage of miR-142-3p zebrafish injected embryos. 3.3. Mir-142-3p Is Expressed during Zebrafish Early Developmental Stages

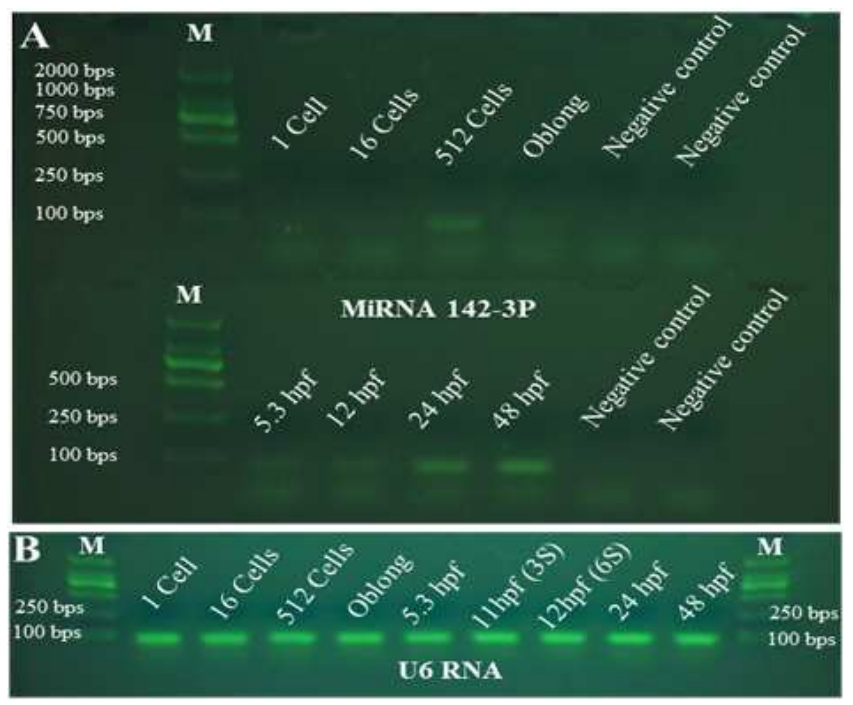

Figure 7. The result of the RT-PCR shows that miR-142-3p was decently expressed in the Zebra fish embryo during early developmental stage (1cell, 16cell, 512cell, Oblong, 5.3hours, 3S, 6s, 1day, 2day) (A) miR-142-3p was expressed maternally from 1 cell stage and decreased its expression afterward till 3 stage and increased its expression significantly on dayl and day2 (B) U6RNA as a control. 
From the RT-PCR (Figure 7) it has been shown that miR-142-3p was decently expressed in the Zebra fish embryo during early developmental stages. Zebra fish embryos were collected at $0 \mathrm{~h}, 1 \mathrm{~h}, 3 \mathrm{~h} 5.3 \mathrm{~h}, 12 \mathrm{~h}, 3 \mathrm{~s}, 1 \mathrm{st}$ day and $2 \mathrm{nd}$ day, and were subjected to total RNA isolation. MiR-142-3p was reverse transcript using miR-142-3p RT primer and amplified by PCR. From the RT result expression levels measurement showed that miR-142-3p was expressed maternally from 1 cell stage and decreased its expression afterward till $3 \mathrm{~s}$ stage and increased its expression significantly on day 1 and day 2 suggesting that miR-142-3p was expressed during early developmental stages.

\subsection{Mir-142-3p is Required for HSC Formation and Definitive Haematopoiesis}

To characterize the function of miR-142-3p during early vertebrate development, we performed the gain-of-function experiments. MiR-142-3p is among the earliest expressed miRNAs detected during zebra fish development. Gain-of-function experiments were performed by injecting miR-142-3p into zebra fish embryos at the one-cell stage followed by assessment of the effects on the HSC tissues by in situ hybridization with the key hematopoietic transcription factor stem cell leukaemia (SCL) that is a hematopoietic cell specific transcription factor and (GATA2) which is expressed in hematopoietic progenitors, including early erythroid cells, mast cells, and megakaryocytes, and also in nonhematopoietic embryonic stem cells (Figure 8) and (PU.1) (Figure 9) which is a hematopoietic cell specific transcription factor for the $(\mathrm{Scl})$ and the erythroid (red blood cell) and megakaryocytic (platelet producing cell) essential development factor GATA1 (Figure10).

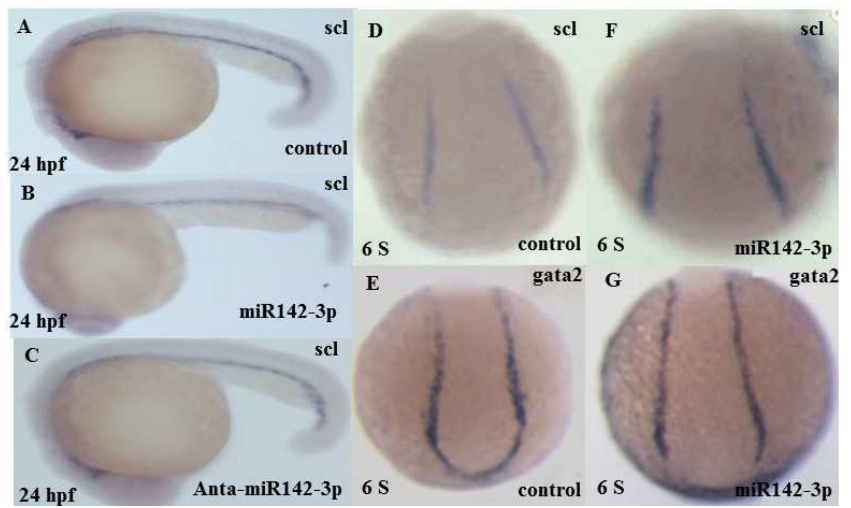

Figure 8. The result of the miR-142-3p gain of function conformation, represses hemo cytoplast (stem cell) formation using (SCL)and (GATA2) marker as indicators (A) lateral view of embryo was non-injected with miR-142-3p at 24 hpf (control) it show a normal blood formation $(B, C)$ lateral views of embryo was injected with miR-142-3p show a diminution in blood formation at $24 \mathrm{hpf}(\mathrm{D})$ embryo was non-injected with miR-142-3p at $12 \mathrm{hpf}$ (control) it show a normal blood formation $(F)$ lateral view of embryo was injected with miR-142-3p at 12 hpf show a diminution in blood formation(E) embryo was non-injected with miR-142-3p at $12 \mathrm{hpf}$ (control) it show a normal blood formation $(G)$ lateral view of embryo was injected with miR-142-3p at 12 hpf show a diminution in blood formation.

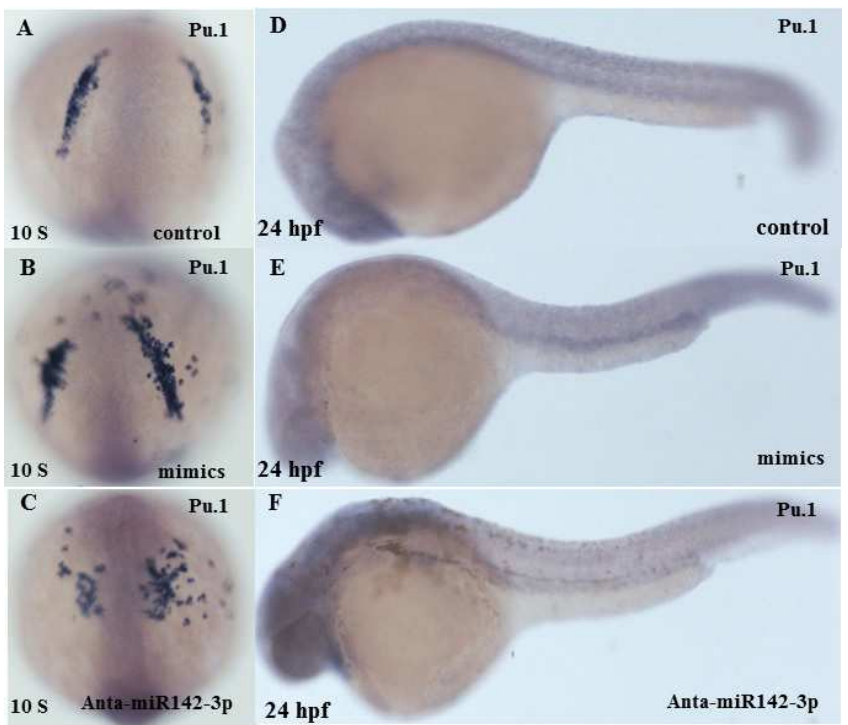

Figure 9. The result of the miR-142-3p gain of function conformation represses myeloid formation using (PU.1) marker as indicator(A) lateral view of embryo was non-injected with miR-142-3p at $12 \mathrm{hpf}$ (control) it show a normal blood formation $(B, C)$ lateral view of embryo was injected with miR-142-3p at 12 hpf show a diminution in blood formation it show a normal blood formation (D) lateral view of embryo was non-injected with miR-142-3p at 24 hpf ( control) it show a normal blood formation (E) embryo was injected with miR-142-3p at 24 hpf show a diminution in blood formation. The result in both $(B)$ and $(E)$ show that no mature macrophages develop, and the neutrophils that develop are aberrantly and incompletely matured.

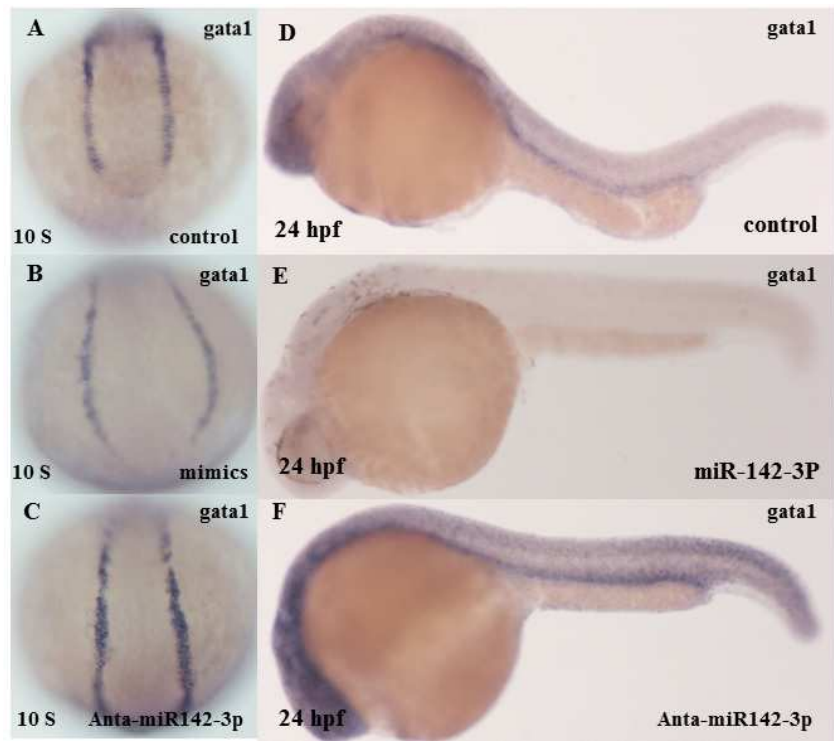

Figure 10. The erythroid (red blood cell) and megakaryocytic (platelet producing cell) essential development factor GATAl gain of function conformation. (A) lateral view of embryo was non-injected with miR-142-3p at $12 \mathrm{hpf}$ (control) it show a normal blood formation $(B, C)$ lateral view of embryo was injected with miR-142-3p at 12 hpf show a diminution in blood formation it show a normal blood formation $(D)$ lateral view of embryo was non-injected with miR-142-3p at 24 hpf (control) it show a normal blood formation (E) embryo was injected with miR-142-3p at 24 hpf show a diminution in blood formation.

It is required in early hematopoietic progenitors for specification of the megakaryocytic lineage and for the (PU.1),Gene disruption of PU.1 results in a cell autonomous 
defect in hematopoietic progenitor cells that manifests as abnormal myeloid and B-lymphoid development. In non-injected controls, it show a normal blood formation but in the injected one, for both markers, miR-142-3p injection caused over $60 \%$ of the embryos to display diminution in blood formation during the 6 somite stage (Figure 8 B\& Figure 9B). A similar phenotype has been observed in the 24 hpf stage (Figure 8 E \&Figure 9E) suggesting possible defects in HSC formation and specification and definitive haematopoiesis.

\subsection{Mir-142-3p is Required for HSC Formation andDefinitive Haematopoiesis}

Through repression of meis1 in zebra fish embryos definitive haematopoiesis generates hematopoietic stem cells (HSCs) [9]. To assess the role of miR-142-3p in definitive haematopoiesis, we analysed the expression of runx 1 and c-myb (markers of HSCs) in zebra fish embryos injected with miR-142-3p mimics by WISH. Injection of miR-142-3p significantly reduced the expression of runx1, c-myb and mpo-1, which suggests that miR-142-3p is required for definitive haematopoiesis (Fig.11).

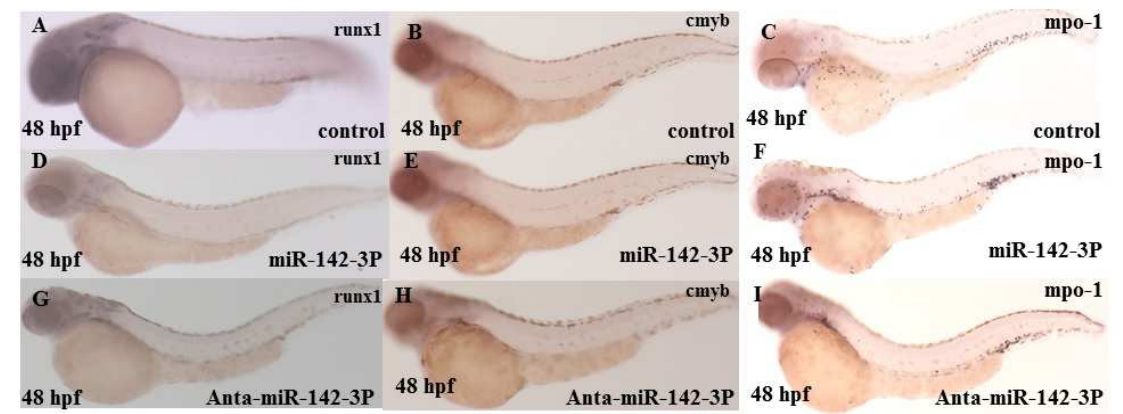

Figure 11. MiR-142-3p regulates primitive hematopoiesis in zebrafish embryos as analyzed by whole-mount in situ hybridization at 24 hpf. (A,B,C) lateral view of embryo was non-injected with miR-142-3p at 24 hpf (control) it show a normal blood formation (D, G) Overexpression of miR-142-3p significantly reduced the expression of runxl at 24 hpf $(E, H)$ Overexpression of miR-142-3p significantly reduced the expression of cmyb. (F, I) Overexpression of miR-142-3p significantly reduced the expression of mpo-1 at $24 \mathrm{hpf}$.

These results suggest that miR-142-3p is required for definitive haematopoiesis through repression of meis 1 in zebra fish embryos. Scl is critical to both primitive and definitive haematopoiesis $[35,36]$. Therefore, we used WISH to analyse the effect of miR-142-3p on expression of scl. Over expression of miR-142-3p dramatically reduced the signal for scl. These results further suggest that miR-142-3p regulates both primitive and definitive haematopoiesis by repressing meis1 expression.

\subsection{MiR-142-3p Regulates Primitive Hematopoiesis, Vascular Development by Repressing the Expression of Meis1 during Embryogenesis in Zebrafish}

Previous studies suggest that meis1 plays an important role in the development of the vasculature $[37,38]$ and that $\mathrm{scl}$ is also involved in endothelial development [35, 39, 40]. Overexpression of meis 1 rescued the effect of miR-142-3p. To further demonstrate the inhibitory effect of miR-142-3p on haematopoiesis, we performed rescue experiments by co-injection of miR-142-3p with meis1 into1-2 cell stage embryos. Therefore, the 3-UTR of meis 1 to create an EGFP

Reporter (Fig.12), a reporter was co-injected with the miR-142-3p into1-2 cell stage embryos and the intensity of the EGFP signal was analysed. Co-injection of miR-142-3p resulted in a statistically significant decrease in EGFP fluorescence compared to co-injection with a negative control, indicating a negative regulatory function of miR-142-3p on meis1, suggesting that the miR-142-3p binding sites are important in its regulation of meis1. MiR-142-3p significantly reduced the expression of EGFP from the reporter with the wild type.

miRs regulate gene expression through either cleavage or translational repression of targeted mRNA [41]. The effect of miR-142-3p on meis1 was more pronounced at the protein level than at the mRNA level, suggesting that miR-142-3p may reduce the stability of meis 1 mRNA and inhibit translation of the meis 1 protein. Taken together, these results indicate that miR-142-3p represses the expression of meis 1 by directly targeting the meis1 3-UTR.

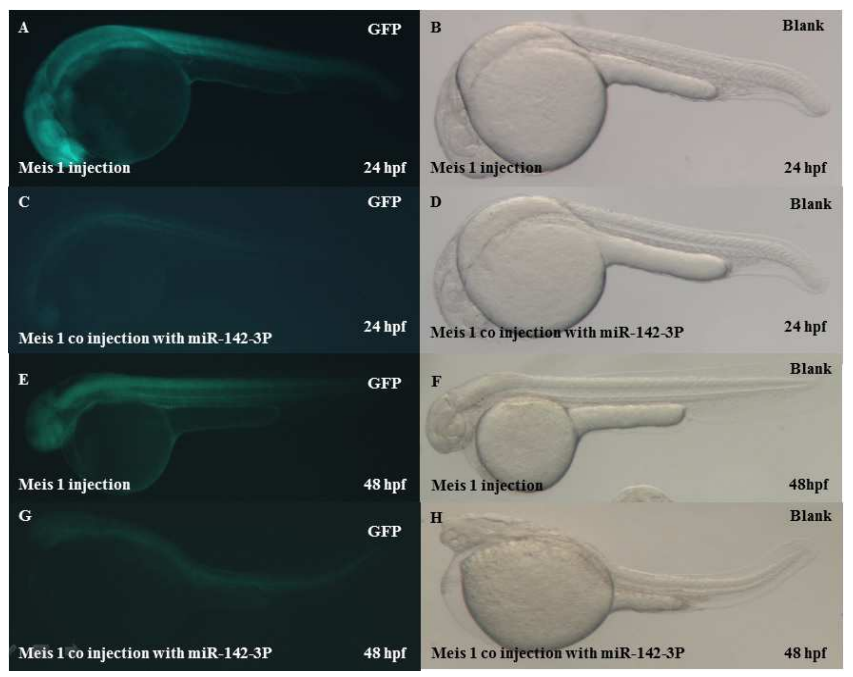

Figure 12. Confirmation of haematopoiesis knock down at 24 hpf \& 48 hpf in zebra fish embryos has co injected with meisl-egfp + miR-142-3p (A,E) green fluorescent pictures show the egfp observation $(C, G)$ co-injected embryo showed reduced in the green color of the meis 1-egfp $(B, D, F, H)$ blank pictures. 


\section{Discussion}

In this study, we demonstrated that miR-142-3p regulated both primitive and definitive haematopoiesis by targeting meis1, regulates Cardiovascular System during zebrafish development. Bioinformatics analysis showed that miR-142-3p regulated meis1 by binding to the 3-UTR of meis1 (Fig. 1). Overexpression of miR-142-3p significantly reduced the number of erythrocytes and the expression of gata1 (the marker for erythroid progenitors). Overexpression of miR-142-3p also significantly reduced the number of myeloid and the expression of pu.1 (the marker for myeloid cells) in zebra fish embryos and the effect was partially rescued by injection of meis1 (Fig. 9).

Similarly, overexpression of miR-142-3p significantly reduced the expression of runx 1 and c-myb (markers for HSCs) and the effect was partially rescued by injection of miRNA of meis1 (Fig. 11). Interestingly, as shown in (Fig. 8), over expression of miR-142-3p decreased expression of scl, GATA2 which is an early upstream regulator of both primitive and definitive haematopoiesis. Together, these results indicate that miR-142-3p is a new molecular determinant required for both primitive and definitive haematopoiesis.

Mechanistically, there regulation of haematopoiesis by miR-142-3p is through repression of meis1. This conclusion is consistent with the spatial and temporal expression profile of miR-142-3p. WISH revealed strong expression [29] supporting its role in haematopoiesis. The regulation may be through miR-142-3p -mediated inhibition of meis1 expression, which may lead to inhibition of scl expression. The meil protein is a 3-amino acid loop extension (TALE) homeodomain protein that was previously identified as playing an important role in primitive and definitive haematopoiesis during zebra fish development [32]. Meis1 was shown to interact with Pbx1 to regulate haematopoiesis [30]. Previous studies suggested that meis1 played an essential role in the development of haematopoiesis and leukemiagenesis, however, the role of meis1 in the hematopoietic signal pathway during zebra fish development is controversial. The production of erythroid was repressed in zebra fish morphants independent of gata1, whereas Pillay et al. suggested that meis1 acted upstream of gata1 to regulate primitive haematopoiesis [32, 34, 42]. Overexpression of meis1 and meis2 greatly enhanced the formation of hematopoietic colonies from embryonic stem cells by maintaining proliferation of hematopoietic progenitor cells and skewing the megakaryocyte-erythroid progenitor to erythroid and toward megakaryocyte development $[43,44]$. Knockdown of meis 1 in zebra fish resulted in a dramatic decrease in the number of erythrocytes [41]. The results above support our conclusion that miR-142-3p represses the expression of meis1 and therefore plays an important role in both primitive and definitive haematopoiesis. Our data showed that overexpression of meis1 partially rescued abnormal phenotypes caused by miR-142-3p. The reason why meis 1 cannot fully rescue abnormal phenotypes caused by miR-142-3p is unknown. One possibility may be that there are other important target genes regulated by miR-142-3p. All of which are involved in haematopoiesis. Overexpression of both meis1 and gata1 (encoding an erythroid-specific transcription factor) fully rescued the abnormal erythropoiesis caused by miR-142-3p, but only partial rescue was observed with meis1 mRNA alone. Bioinformatics analysis did not identify any potential miR-142-3p binding site on the gata1 mRNA (data not shown). It is interesting to note that meis1 was shown to act upstream of gata1 to regulate primitive haematopoiesis [34]. Thus, miR-142-3p may indirectly regulate gata1 expression through regulating expression of meis1. This may explain why over-expression of gatal partially rescued the abnormal hematopoietic phenotypes associated with miR-142-3p overexpression [45]. Moreover, miR-142-3p may indirectly regulate gata1 expression through regulating expression of other regulatory genes. This may explain why overexpression of both meis1 and gatal fully rescue the defects by miR-142-3p. Interestingly, gata1 was shown to directly activate the transcription of miR-142-3p [46], which forms a loop signalling pathway involved in haematopoiesis. Pillay et al. found that a meis1 morpholino increased WISH signals for pu.1 and 1-plastin (downstream of pu.1) at 24 hpf [34]. In contrast, Cvejic et al. reported that two different meis1 morpholinos dramatically decreased 1-plastin signal at 28 hpf by 3.3-fold [32]. The reason for the different observations is unknown. However, our data are consistent with the finding by Cvejic et al., i.e. overexpression of miR-142-3p led to decreased expression of meis1 and decreased WISH signal for pu.1. Therefore, our data support the notion that miR-142-3p mediated pu.1 reduction is through regulation of meis1. On the other hand, because miR-142-3p may regulate expression of other downstream target genes, we cannot exclude the possibility that miR-142-3p mediated pu.1 reduction may also involve other target genes of miR-142-3p . In conclusion, we found that miR-142-3p is required for haematopoiesis development by down-regulating the expression level of meis1. This study therefore identifies a new regulator for concerted hematopoietic and vascular development.

\section{Acknowledgements}

We thank Dr.Haibo jia and Dr .A. S. Ishtiaq Ahmed in Huazhong University of Science \& Technology, Dr. Zongbin Cui and his team in the Chinese Academy of Science-Institute of Hydrobiology (Wuhan), for technical assistance and helpful discussion.

\section{References}

[1] Hogan, B.M., et al., Specification of the primitive myeloid precursor pool requires signaling through Alk8 in zebrafish. Curr Biol, 2006. 16(5): p. 506-11.

[2] Rhodes, J., et al., Interplay of pu.1 and gata1 determines myelo-erythroid progenitor cell fate in zebrafish. Dev Cell, 2005. 8(1): p. 97-108.

[3] Yamaguchi, Y., et al., Forced GATA-1 expression in the murine myeloid cell line M1: induction of c-Mpl expression and megakaryocytic/erythroid differentiation. Blood, 1998. 91(2): p. $450-7$. 
[4] Yeh, J.R., et al., AML1-ETO reprograms hematopoietic cell fate by downregulating scl expression. Development, 2008. 135(2): p. 401-10.

[5] Du, L., et al., Rumba and Haus3 are essential factors for the maintenance of hematopoietic stem/progenitor cells during zebrafish hematopoiesis. Development, 2011. 138(4): p. 619-29.

[6] Warga, R.M., D.A. Kane, and R.K. Ho, Fate mapping embryonic blood in zebrafish: multi- and unipotential lineages are segregated at gastrulation. Dev Cell, 2009. 16(5): p. 744-55.

[7] Yuan, H., et al., Sumoylation of CCAAT/enhancer-binding protein alpha promotes the biased primitive hematopoiesis of zebrafish. Blood, 2011. 117(26): p. 7014-20.

[8] Ellett, F. and G.J. Lieschke, Zebrafish as a model for vertebrate hematopoiesis. Curr Opin Pharmacol, 2010. 10(5): p. 563-70.

[9] Sood, R. and P. Liu, Novel insights into the genetic controls of primitive and definitive hematopoiesis from zebrafish models. Adv Hematol, 2012. 2012: p. 830703.

[10] Palis, J. and G.B. Segel, Developmental biology of erythropoiesis. Blood Rev, 1998. 12(2): p. 106-14.

[11] Ma, D., et al., The identification and characterization of zebrafish hematopoietic stem cells. Blood, 2011. 118(2): p. 289-97.

[12] Gardiner, M.R., et al., A global role for zebrafish klf4 in embryonic erythropoiesis. Mech Dev, 2007. 124(9-10): p. 762-74.

[13] Amali, A.A., et al., Zebrafish hoxd4a acts upstream of meis1.1 to direct vasculogenesis, angiogenesis and hematopoiesis. PLoS One, 2013. 8(3): p. e58857.

[14] Juarez, M.A., et al., Distinct roles for SCL in erythroid specification and maturation in zebrafish. J Biol Chem, 2005. 280(50): p. 41636-44.

[15] Liang, D., et al., Retinoic acid signaling plays a restrictive role in zebrafish primitive myelopoiesis. PLoS One, 2012. 7(2): p. e30865.

[16] Zhao, Y. and D. Srivastava, A developmental view of microRNA function. Trends Biochem Sci, 2007. 32(4): p. 189-97.

[17] Lee, R.C., R.L. Feinbaum, and V. Ambros, The C. elegans heterochronic gene lin-4 encodes small RNAs with antisense complementarity to lin-14. Cell, 1993. 75(5): p. 843-54.

[18] Bruchova, H., et al., Regulated expression of microRNAs in normal and polycythemia vera erythropoiesis. Exp Hematol, 2007. 35(11): p. 1657-67.

[19] Bruchova, H., M. Merkerova, and J.T. Prchal, Aberrant expression of microRNA in polycythemia vera. Haematologica, 2008. 93(7): p. 1009-16.

[20] Chen, S.Y., et al., The genomic analysis of erythrocyte microRNA expression in sickle cell diseases. PLoS One, 2008. 3(6): p. e2360.

[21] Westerfield, M., The Zebrafish book : a guide for the laboratory use of zebrafish (Brachydanio rerio). 1993, Eugene. Or: University of Oregon Press. 1 v. (various pagings).

[22] Chen, D., et al., Functional characterization of
Klippel-Trenaunay syndrome gene AGGF1 identifies a novel angiogenic signaling pathway for specification of vein differentiation and angiogenesis during embryogenesis. Hum Mol Genet, 2013. 22(5): p. 963-76.

[23] Zhou, B., et al., MicroRNA-503 targets FGF2 and VEGFA and inhibits tumor angiogenesis and growth. Cancer Lett, 2013. 333(2): p. 159-69.

[24] Du, T.T., et al., Experimental validation and complexity of miRNA-mRNA target interaction during zebrafish primitive erythropoiesis. Biochem Biophys Res Commun, 2009. 381(4): p. 688-93.

[25] Grabher, C., et al., Zebrafish microRNA-126 determines hematopoietic cell fate through c-Myb. Leukemia, 2011. 25(3): p. $506-14$

[26] Starczynowski, D.T., et al., Identification of miR-142-3p and miR-146a as mediators of the $5 \mathrm{q}$ - syndrome phenotype. Nat Med, 2010. 16(1): p. 49-58.

[27] Lagos-Quintana, M., et al., New microRNAs from mouse and human. RNA, 2003. 9(2): p. 175-9.

[28] Pase, L., et al., miR-451 regulates zebrafish erythroid maturation in vivo via its target gata2. Blood, 2009. 113(8): $p$. 1794-804.

[29] Fu, Y.F., et al., Mir-144 selectively regulates embryonic alpha-hemoglobin synthesis during primitive erythropoiesis. Blood, 2009. 113(6): p. 1340-9.

[30] Thisse, C. and B. Thisse, High-resolution in situ hybridization to whole-mount zebrafish embryos. Nat Protoc, 2008. 3(1): p. 59-69.

[31] Azzouzi, I., M. Schmugge, and O. Speer, MicroRNAs as components of regulatory networks controlling erythropoiesis. Eur J Haematol, 2012. 89(1): p. 1-9.

[32] Cvejic, A., et al., The role of meis 1 in primitive and definitive hematopoiesis during zebrafish development. Haematologica, 2011. 96(2): p. 190-8.

[33] Hu, Y.L., et al., HOXA9 modulates its oncogenic partner Meis1 to influence normal hematopoiesis. Mol Cell Biol, 2009. 29(18): p. 5181-92.

[34] Pillay, L.M., et al., The Hox cofactors Meis1 and Pbx act upstream of gata1 to regulate primitive hematopoiesis. Dev Biol, 2010. 340(2): p. 306-17.

[35] Dooley, K.A., A.J. Davidson, and L.I. Zon, Zebrafish scl functions independently in hematopoietic and endothelial development. Dev Biol, 2005. 277(2): p. 522-36.

[36] Qian, F., et al., Distinct functions for different scl isoforms in zebrafish primitive and definitive hematopoiesis. PLoS Biol, 2007. 5(5): p. e132.

[37] Azcoitia, V., et al., The homeodomain protein Meis1 is essential for definitive hematopoiesis and vascular patterning in the mouse embryo. Dev Biol, 2005. 280(2): p. 307-20.

[38] Minehata, K., A. Kawahara, and T. Suzuki, meis1 regulates the development of endothelial cells in zebrafish. Biochem Biophys Res Commun, 2008. 374(4): p. 647-52.

[39] Patterson, L.J., M. Gering, and R. Patient, Scl is required for dorsal aorta as well as blood formation in zebrafish embryos. Blood, 2005. 105(9): p. 3502-11. 
[40] Van Handel, B., et al., Scl represses cardiomyogenesis in prospective hemogenic endothelium and endocardium. Cell, 2012. 150(3): p. 590-605.

[41] Rosen, J.N., M.F. Sweeney, and J.D. Mably, Microinjection of zebrafish embryos to analyze gene function. J Vis Exp, 2009(25)

[42] Sangokoya, C., M.J. Telen, and J.T. Chi, microRNA miR-144 modulates oxidative stress tolerance and associates with anemia severity in sickle cell disease. Blood, 2010. 116(20): p. 4338-48.

[43] Cai, M., et al., Dual actions of Meis1 inhibit erythroid progenitor development and sustain general hematopoietic cell proliferation. Blood, 2012. 120(2): p. 335-46.

[44] Moskow, J.J., et al., Meis1, a PBX1-related homeobox gene involved in myeloid leukemia in BXH-2 mice. Mol Cell Biol, 1995. 15(10): p. 5434-43.

[45] Su, Z., et al., MiR-144 regulates hematopoiesis and vascular development by targeting meis1 during zebrafish development. Int J Biochem Cell Biol, 2014. 49: p. 53-63.

[46] Dore, L.C., et al., A GATA-1-regulated microRNA locus essential for erythropoiesis. Proc Natl Acad Sci U S A, 2008. 105(9): p. 3333-8. 